\title{
Anti-atherosclerotic Activity of Triacsin C, an Acyl-CoA Synthetase Inhibitor
}

\author{
Daisuke Matsuda, Ichiji Namatame, Taichi Ohshiro, Shun Ishibashi, Satoshi Ōmura, \\ Hiroshi Tomoda
}

Received: March 12, 2008 / Accepted: April 21, 2008

(C) Japan Antibiotics Research Association

\begin{abstract}
As previously reported, triacsin C, a selective inhibitor of acyl-CoA synthetase, inhibited the synthesis of cholesteryl ester and triacylglycerol in mouse peritoneal macrophages, leading to a reduction of lipid droplets. Therefore, the in vivo efficacy was studied. Low-density lipoprotein receptor-knockout $\left(\mathrm{LDLR}^{-/}\right)$mice were fed a high cholesterol diet $(0.15 \%)$ for two months to measure the atherogenic areas of the hearts and aortas. When triacsin C was orally administered $(10 \mathrm{mg} / \mathrm{kg} / \mathrm{day})$, the atherosclerotic areas were significantly reduced by $86 \%$ in aorta and $36 \%$ in hearts. The results strongly suggested that triacsin $\mathrm{C}$ shows anti-atherogenic activity by inhibiting acyl-CoA synthetase activity.
\end{abstract}

Keywords acyl-CoA synthetase, atherosclerosis, in vivo, low-density lipoprotein receptor-knock out mice, triacsin C

\section{Introduction}

In the early stage of atherosclerosis, macrophages penetrate into the intima, efficiently take up modified low density lipoprotein (LDL), store cholesterol and fatty acid as the respective forms of cholesteryl ester (CE) and triacylglycerol (TG) in cytosolic lipid droplets, and are converted to foam cells, leading to the development of atherosclerosis in the arterial wall. Therefore, inhibitors of macrophage lipid droplet formation would be expected to retard the progression of atherosclerosis [1 4].

A cell-based assay was established to observe lipid droplet formation using primary mouse peritoneal macrophages [5] as a model of macrophage-derived foam cell formation in the artery. When macrophages are cultured with phosphatidylserine-containing liposomes, the liposomes are recognized by scavenger receptors and metabolized in a way similar to the natural ligand-modified LDL [6], resulting in the accumulation of lipid droplets in the cytosol. In the morphological assays, lipid droplet formation was observed microscopically after staining lipid droplets with oil red $\mathrm{O}$. In the biochemical assay, the amounts of $\left[{ }^{14} \mathrm{C}\right] \mathrm{CE}$ and $\left[{ }^{14} \mathrm{C}\right] \mathrm{TG}$ produced in the lipid droplets were measured by the addition of $\left[{ }^{14} \mathrm{C}\right]$ oleic acid to the culture medium.

Taking advantage of this model, we found that triacsins $\mathrm{A}$ and $\mathrm{C}$, well-known inhibitors of long chain acyl-CoA synthetase (ACS), inhibited the synthesis of $\left[{ }^{14} \mathrm{C}\right] \mathrm{CE}\left(\mathrm{IC}_{50}\right.$ values; 0.18 and $0.19 \mu \mathrm{M}$, respectively) and $\left[{ }^{14} \mathrm{C}\right] \mathrm{TG}\left(\mathrm{IC}_{50}\right.$ values; 0.16 and $0.10 \mu \mathrm{M}$, respectively), and caused the complete inhibition of lipid droplet synthesis in mouse macrophages [7]. Triacsins A to D were isolated from the culture filtrate of Streptomyces sp. SK-1894 and consisted of 11-carbon alkenyl chains with a common triazenol moiety at their termini $[8,9]$. On the basis of its unique target of inhibition, triacsins have been utilized in many
D. Matsuda, T. Ohshiro, H. Tomoda (Corresponding author): School of Pharmacy, Kitasato University, 5-9-1 Shirokane, Minato-ku, Tokyo 108-8641, Japan, E-mail: tomodah@pharm.kitasato-u.ac.jp
I. Namatame, S. Ōmura: Kitasato Institute for Life Sciences and Graduate School of Infection Control Sciences, Kitasato University, 5-9-1 Shirokane, Minato-ku, Tokyo 108-8641, Japan

S. Ishibashi: Division of Endocrinology and Metabolism, Jichi Medical University, 3311-1 Yakushiji, Shimotsuke, Tochigi 3290498, Japan 
studies to investigate the function of ACS in lipid metabolism [5, 10 13]. These findings prompted us to study the in vivo efficacy of triacsins in an atherosclerogenic mouse model. In this study, we show anti-atherosclerotic activity of triacsin $\mathrm{C}$ in $\mathrm{LDLR}^{-1-}$ mice.

\section{Materials and Methods}

\section{Materials}

Triacsin C (Fig. 1) was purified from a culture broth of Streptomyces sp. SK-1894 by an established method [9]. Oil red O was purchased from SIGMA. Sudan IV was obtained from Wako Pure Chemical Industries (Osaka, Japan). Low-density lipoprotein receptor-knockout $\left(\mathrm{LDLR}^{-1-}\right.$ ) mice on a C57BL/6 background were purchased from The Jackson Laboratory (ME, USA).

\section{In Vivo Anti-atherosclerotic Activity}

$\mathrm{LDLR}^{-1-}$ mice were treated as previously described [14]. Eight-week old mice were fed $0.15 \%$ cholesterolsupplemented diets (CLEA Japan, Tokyo, Japan), and $0.05 \%$ sodium carboxy methyl cellulose with or without triacsin $\mathrm{C}(10 \mathrm{mg} / \mathrm{kg}$ of body weight) was administered orally every day for two months. Blood was collected from the retroorbital venous plexus at zero, one, and two months. The levels of glucose, total cholesterol, TG, and free fatty acid in plasma were measured using an Advantage II (Roche Diagnostics), Determiner TC555 kit (Kyowa Medex, Tokyo, Japan), Triglyceride G-test kit (Wako Pure Chemicals, Osaka, Japan), and NEFA C-test kit (Wako Pure Chemicals), respectively.

For atherosclerotic lesion analyses, whole aortas were stained with Sudan IV, and cross sections of the proximal aorta were prepared and stained with oil red $\mathrm{O}$. The hearts were perfused with phosphate-buffered saline containing $3.0 \%$ formalin, were embedded in OCT compound (Sakura Tissue-Tek, Tokyo, Japan), and 6- $\mu$ m-thick serial sections were cut by using a Cryostat (Leica, Tokyo, Japan). Four sections, each separated by $60 \mu \mathrm{m}$, were counter-stained with oil red $\mathrm{O}$ and hematoxylin and used to evaluate the lesions. Images of the sections were captured with a digital camera (CoolSnap Photometrics, Olympus, Tokyo, Japan) mounted on a light microscope (Vanox-S model, Olympus). Image analysis was performed by using Photoshop 6.0 software (Adobe).

$$
\text { N-N=N-OH }
$$

Fig. 1 Structure of triacsin $C$.

\section{Results and Discussion}

After two-month feeding with a high cholesterol diet, $\mathrm{LDLR}^{-1-}$ mice developed atherosclerotic lesions in the aortas and the hearts (Fig. 2A and 2C, respectively). The atherosclerotic area was calculated to be $6.27 \%$ in the aortas and $10.2 \mu \mathrm{m}^{2}$ in the hearts. When triacsin $\mathrm{C}$ was administered orally $(10 \mathrm{mg} / \mathrm{kg} /$ day $)$ for two months, the atherosclerotic areas decreased to $0.90 \%(P<0.05)$ in the aortas (Fig. 2B) and to $6.5 \mu \mathrm{m}^{2}(P<0.05)$ in the hearts (Fig. 2D), indicating $86 \%$ (Fig. 2E) and $36 \%$ reduction (Fig. 2F), respectively. The administration concentration of triacsin $\mathrm{C}$
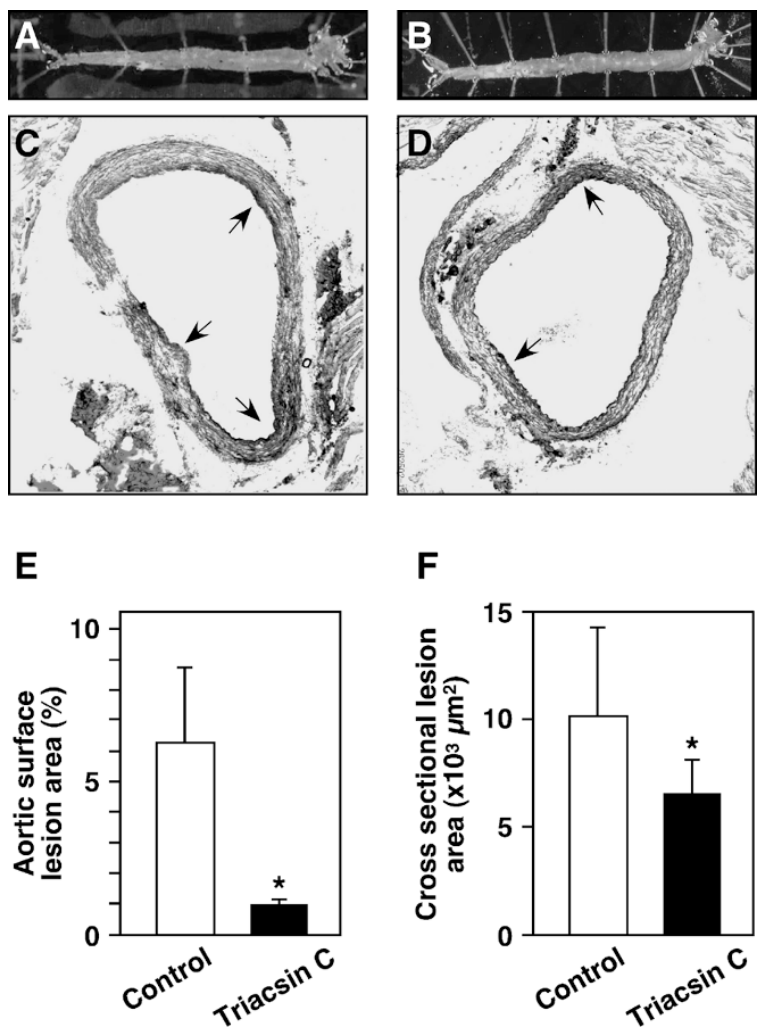

Fig. 2 Effect of triacsin $\mathrm{C}$ on aortic atherosclerosis of $\mathrm{LDLR}^{-1-}$ mice.

$\mathrm{LDLR}^{-/-}$mice were fed $0.15 \%$ cholesterol-supplemented diets with or without triacsic C $(10 \mathrm{mg} / \mathrm{kg} /$ day $)$ for two months $(n=10$, each group). (A and B) Pinned-out aortas showing sudan-IVstained lesions from $\mathrm{LDLR}^{-1-}$ mice that received $0.05 \%$ sodium CM-cellulose containing triacsin C $(10 \mathrm{mg} / \mathrm{kg} /$ day orally for two months) (B) and only $0.05 \%$ sodium CM-cellulose (control) (A). (C and D) Cross sections of proximal aortic roots of hearts showing oil-red-O-staining lesions in $\mathrm{LDLR}^{-1-}$ mice treated with triacsin C (D) and control (C). Comparison of the size of whole aorta surface (E) for A and B, and cross-sectional (F) lesions for C and D between the control and triacsin $\mathrm{C}$-treated groups. Arrows indicate the atherosclerotic lesion. Bars indicate mean and error bars represent $\pm \mathrm{SD} ;{ }^{*}, P<0.05$. 
A

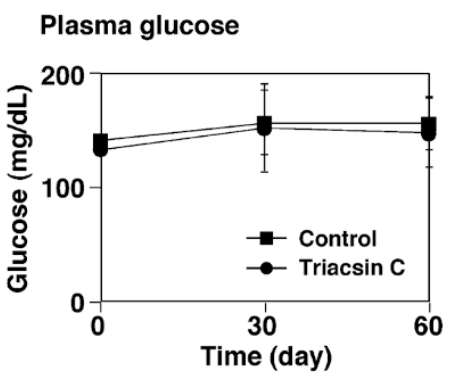

C

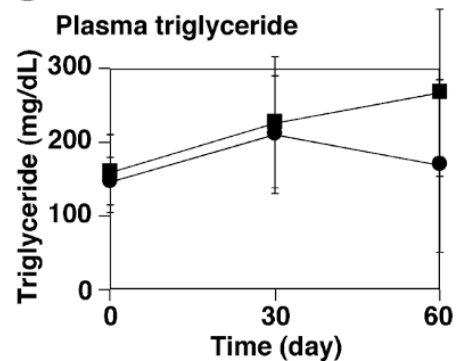

B

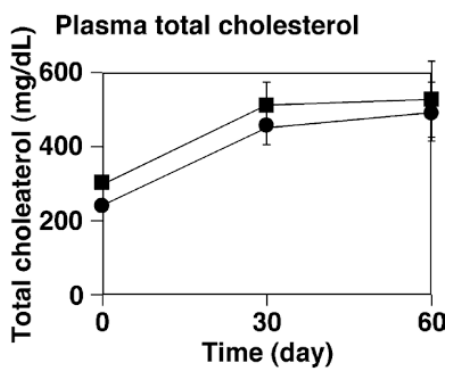

D

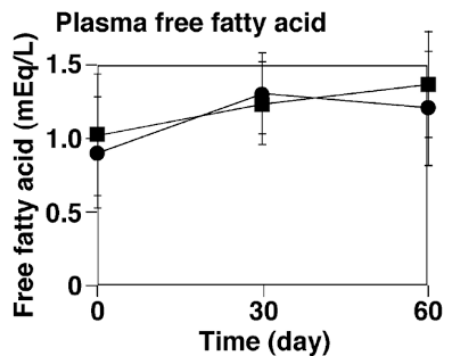

Fig. 3 Effect of triacsin $C$ on plasma TG, plasma cholesterol, plasma free fatty acid, and plasma glucose concentration.

Plasma concentration of TG(A), cholesterol (B), free fatty acid (C), or glucose (D) of $\mathrm{LDLR}^{-1-}$ mice treated with triacsin C (-) or vehicle alone (घ) for two months. Each concentration was measured as shown in the "Materials and Methods". Bars indicate mean and error bars represent $\pm S D$.

$(10 \mathrm{mg} / \mathrm{kg} / \mathrm{day})$ was set up by referring to the case of beauveriolides I and III. They inhibited the accumulation of $\mathrm{CE}$ in mouse peritoneal macrophages with $\mathrm{IC}_{50}$ values of 0.78 and $0.41 \mu \mathrm{M}$, respectively, and exerted antiatherosclerotic activity by administering $25 \sim 50 \mathrm{mg} / \mathrm{kg} / \mathrm{day}$ under the same conditions [14].

There were no significant differences in the levels of glucose, total cholesterol and free fatty acid in the plasma during the two-month experiments, while the TG levels decreased to $70 \%$ of the control on day 60 (Fig. 3). Nestel et al. reported that cis-9,trans-11 isomer of conjugated linoleic acid reduced plasma TG but did not suppress aortic atherosclerosis [16]. Furthermore, triacsin $C$ showed no toxic effects on various tissues such as adrenal tissues and livers, showed no diarrhea in mice, and only a slight weight loss was observed (Fig. 4).

It might be that triacsin $\mathrm{C}$, an ACS inhibitor, directly affects macrophage-derived foam cell formation in the aortas and hearts of $\mathrm{LDL}^{-/-}$mice by inhibition of $\mathrm{CE}$ and TG synthesis in macrophages. Furthermore, triacsin $\mathrm{C}$ was reported to show anti-atherogenic activity to affect the several atherogenic factors. Triacsin $\mathrm{C}$ was reported to inhibit the generation of nitric oxide and superoxide anion $[13,18]$, to induce the downregulation of leptin $[19,20]$ and the hydrolysis of TG [21], and to inhibit the secretion

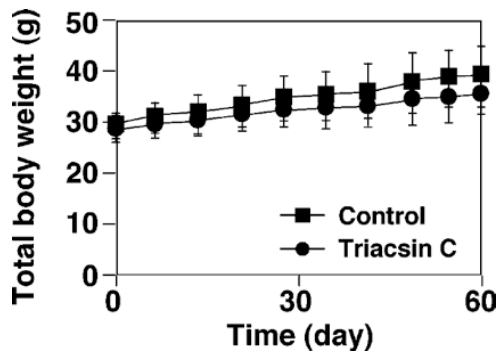

Fig. 4 Effect of triacsin $\mathrm{C}$ on the body weight of $\mathrm{LDL}^{-/-}$ mice.

Body weight of $\mathrm{LDLR}^{-1-}$ mice treated with triacsin $\mathrm{C}(\bullet)$ or vehicle alone (ם) for two months. Bars indicate mean and error bars represent \pm SD.

of apolipoprotein B $[22,23]$. We considered that the accumulation of these several effect induced the suppression of atherosclerosis.

Acknowledgement This work was supported by the Program for Promotion of Fundamental Studies in Health Sciences of the National Institute of Biomedical Innovation (NIBIO) and by a grant-in-aid for Scientific Research on Priority Areas 16073215 from the Ministry of Education, Culture, Sports, Science and Technology, Japan. 
References

1. Goldstein JL, Ho YK, Basu SK, Brown MS. Binding site on macrophages that mediates uptake and degradation of acetylated low density lipoprotein, producing massive cholesterol deposition. Proc Natl Acad Sci USA 76: 333-337 (1979)

2. Brown MS, Goldstein JL, Krieger M, Ho YK, Anderson RG. Reversible accumulation of cholesteryl esters in macrophages incubated with acetylated lipoproteins. J Cell Biol 82: 597-613 (1979)

3. Schaffner T, Taylor K, Bartucci EJ, Fischer-Dzoga K, Beeson JH, Glagov S, Wissler RW. Arterial foam cells with distinctive immunomorphologic and histochemical features of macrophages. Am J Pathol 100: 57-80 (1980)

4. Gerrity RG. The role of the monocyte in atherogenesis: I. Transition of blood-borne monocytes into foam cells in fatty lesions. Am J Pathol 103: 181-190 (1981)

5. Morikawa Y, Hinata S, Tomoda H, Goto T, Nakai M, Aizawa C, Tanaka H, Ōmura S. Complete inhibition of human immunodeficiency virus Gag myristoylation is necessary for inhibition of particle budding. J Biol Chem 271: 2868-2873 (1996)

6. Nishikawa K, Sato Y, Arai H, Inoue K. Mobilization of acyl chains from endogenous cellular phospholipids into cholesteryl esters during foam-cell formation in mouse peritoneal macrophages. Biochim Biophys Acta 1169: 257-263 (1993)

7. Namatame I, Tomoda $\mathrm{H}$, Arai $\mathrm{H}$, Inoue $\mathrm{K}$, Ōmura $\mathrm{S}$. Complete inhibition of mouse macrophage-derived foam cell formation by triacsin C. J Biochem 125: 319-327 (1999)

8. Tomoda H, Igarashi K, Ōmura S. Inhibition of acyl-CoA synthetase by triacsins. Biochim Biophys Acta 921: 595-598 (1987)

9. Ōmura S, Tomoda H, Xu QM, Takahashi Y, Iwai Y. Triacsins, new inhibitors of acyl-CoA synthetase produced by Streptomyces sp. J Antibiot 39: 1211-1218 (1986)

10. Pfanner N, Orci L, Glick BS, Amherdt M, Arden SR, Malhotra V, Rothman JE. Fatty acyl-coenzyme A is required for budding of transport vesicles from Golgi cisternae. Cell 59: 95-102 (1989)

11. Tomoda H, Igarashi K, Cyong JC, Ōmura S. Evidence for an essential role of long chain acyl-CoA synthetase in animal cell proliferation. Inhibition of long chain acyl-CoA synthetase by triacsins caused inhibition of Raji cell proliferation. J Biol Chem 266: 4214-4219 (1991)

12. Wu X, Sakata N, Lui E, Ginsberg HN. Evidence for a lack of regulation of the assembly and secretion of apolipoprotein B-containing lipoprotein from HepG2 cells by cholesteryl ester. J Biol Chem 269: 12375-12382 (1994)

13. Korchak HM, Kane LH, Rossi MW, Corkey BE. Long chain acyl coenzyme A and signaling in neutrophils. An inhibitor of acyl coenzyme A synthetase, triacsin C, inhibits superoxide anion generation and degranulation by human neutrophils. J Biol Chem 269: 30281-30287 (1994)

14. Namatame I, Tomoda H, Ishibashi S, Ōmura $\mathrm{S}$. Antiatherogenic activity of fungal beauveriolides, inhibitors of lipid droplet accumulation in macrophages. Proc Natl Acad Sci USA 101: 737-742 (2004)

15. Matsuda D, Tomoda H. DGAT inhibitors for obesity. Curr Opin Investig Drugs 8: 836-841 (2007)

16. Nestel P, Fujii A, Allen T. The cis-9,trans-11 isomer of conjugated linoleic acid (CLA) lowers plasma triglyceride and raises HDL cholesterol concentrations but does not suppress aortic atherosclerosis in diabetic apoE-deficient mice. Atherosclerosis 189: 282-287 (2006)

17. Igal RA, Coleman RA. Acylglycerol recycling from triacylglycerol to phospholipid, not lipase activity, is defective in neutral lipid storage disease fibroblasts. J Biol Chem 271: 16644-16651 (1996)

18. Weis MT, Crumley JL, Young LH, Stallone JN. Inhibiting long chain fatty Acyl CoA synthetase increases basal and agonist-stimulated NO synthesis in endothelium. Cardiovasc Res 63: 338-346 (2004)

19. Shintani M, Nishimura $H$, Yonemitsu S, Masuzaki $H$, Ogawa Y, Hosoda K, Inoue G, Yoshimasa Y, Nakao K. Downregulation of leptin by free fatty acids in rat adipocytes: effects of triacsin C, palmitate, and 2bromopalmitate. Metabolism 49: 326-330 (2000)

20. Arai T, Kawakami Y, Matsushima T, Okuda Y, Yamashita K. Intracellular fatty acid downregulates ob gene expression in 3T3-L1 adipocytes. Biochem Biophys Res Commun 297: 1291-1296 (2002)

21. Lada AT, Willingham MC, St. Clair RW. Triglyceride depletion in THP-1 cells alters cholesteryl ester physical state and cholesterol efflux. J Lipid Res 43: 618-628 (2002)

22. Wu X, Sakata N, Dixon J, Ginsberg HN. Exogenous VLDL stimulates apolipoprotein B secretion from HepG2 cells by both pre- and post-translational mechanisms. J Lipid Res 35: 1200-1210 (1994)

23. Pan M, Liang JS, Fisher EA, Ginsberg HN. The late addition of core lipids to nascent apolipoprotein B100, resulting in the assembly and secretion of triglyceride-rich lipoproteins, is independent of both microsomal triglyceride transfer protein activity and new triglyceride synthesis. J Biol Chem 277: 4413-4421 (2002) 